\title{
Aerospace Applications of Optimization under Uncertainty
}

\author{
Sharon Padula, Clyde Gumbert, and Wu Li \\ NASA Langley Research Center
}

\begin{abstract}
The Multidisciplinary Optimization (MDO) Branch at NASA Langley Research Center develops new methods and investigates opportunities for applying optimization to aerospace vehicle design. This paper describes MDO Branch experiences with three applications of optimization under uncertainty: (1) improved impact dynamics for airframes, (2) transonic airfoil optimization for low drag, and (3) coupled aerodynamic/structures optimization of a 3-D wing. For each case, a brief overview of the problem and references to previous publications are provided. The three cases are aerospace examples of the challenges and opportunities presented by optimization under uncertainty. The present paper will illustrate a variety of needs for this technology, summarize promising methods, and uncover fruitful areas for new research.
\end{abstract}

\section{Introduction}

In 2002, a team of researchers at NASA Langley completed a survey of the state of the art in uncertainty quantification (UQ) and in robust or reliability-based design of engineering systems [1]. The team initiated a research effort called uncertainty-based methods (UBM) and highlighted the need for improved computational and experimental methods applied to multidisciplinary aerospace vehicle design problems. One of the areas recommended for further research was optimization under uncertainty. The present paper summarizes recent experiences with three separate optimization applications. The first application optimizes a simplified airframe finite element model (FEM) for improved crashworthiness. The second optimization reduces the drag of an airfoil while maintaining lift and treats the transonic Mach number as an uncertain variable. The third application improves the performance of a flexible wing assuming small uncertainty in the geometric variables and including constraints on payload, compliance, and trimability.

The three applications have similarities. Each has a cost function and/or constraints that are sensitive to uncertain parameters. All of the design variables are continuous, and the numerical simulations are nonlinear and computationally expensive. All three applications show the value of optimization under uncertainty compared with conventional deterministic optimization.
The applications are also different in a number of ways. For example, one has a large number of design variables while the others have only a few variables. The objective can be to optimize the expected value of performance or to limit the probability of failure. The applications use several different UQ methods and different optimization approaches. The differences in the applications allow the authors to draw general conclusions about the adequacy of existing techniques for aerospace applications and to compile a prioritized list of enabling technologies that require future study.

\section{Aircraft impact dynamics}

The first example of optimization research is motivated by the competing goals of reducing the weight of a small aircraft while improving its safety and crashworthiness. One interesting concept is to design the airframe itself to absorb some of the energy from impact. This idea is investigated in [2] using a simplified 2-D beam model and in [3] using a higher fidelity 3-D model. The 2-D model, sketched in Fig. 1, has 166 elements and uses beams and lumped masses to capture the basic physics of an aluminum fuselage section that is dropped vertically. Studying the 2-D model uncovers both the capabilities and limitations of UBM for high-fidelity impact dynamic applications.

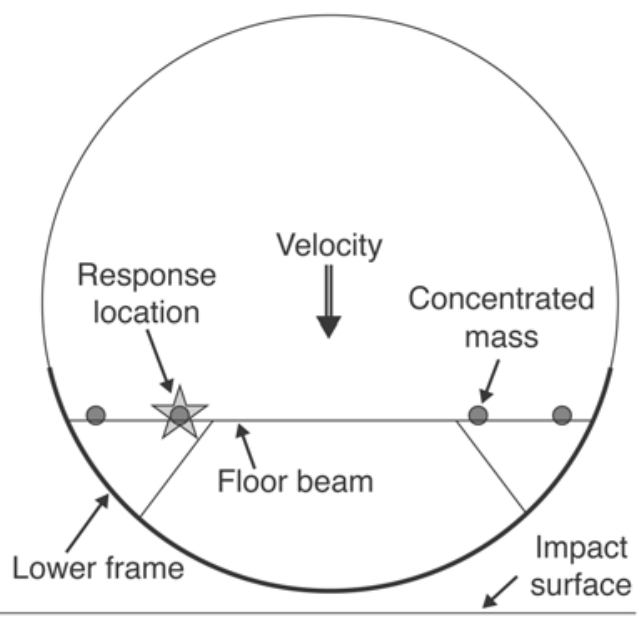

Figure 1. Schematic of the 2-D airframe FEM 
A demonstration problem is defined in [2] to investigate optimization under uncertainty. The design variables $v$ and uncertain parameters $u$ are identified in Table 1 .

\section{Table 1. Design variables and uncertain variables}

\begin{tabular}{|c|c|c|c|c|}
\hline \multirow{2}{*}{$\begin{array}{c}\mathrm{I} / \mathrm{O} \\
\text { symbol }\end{array}$} & \multirow{2}{*}{ Definition } & \multirow{2}{*}{ Mean } & \multicolumn{2}{|c|}{ Bounds } \\
\hline & & & Lower & Upper \\
\hline$v_{1}$ & $\begin{array}{l}\text { Floor Beam } \\
\text { Area }\end{array}$ & $3.00 \mathrm{e}-4$ & $2.42 \mathrm{e}-4$ & $3.58 \mathrm{e}-4$ \\
\hline$v_{2}$ & $\begin{array}{l}\text { Floor Beam } \\
\text { Inertia }\end{array}$ & $4.77 \mathrm{e}-7$ & $4.48 \mathrm{e}-7$ & $5.06 \mathrm{e}-8$ \\
\hline$v_{3}$ & $\begin{array}{l}\text { Lower Frame } \\
\text { Area }\end{array}$ & $3.50 \mathrm{e}-4$ & $2.92 \mathrm{e}-4$ & $4.08 \mathrm{e}-4$ \\
\hline$u_{1}$ & Yield Strength & $3.50 \mathrm{e} 8$ & $2.92 \mathrm{e} 8$ & $4.08 \mathrm{e} 8$ \\
\hline$u_{2}$ & Impact Velocity & -7.00 & -8.15 & -5.85 \\
\hline$u_{3}$ & Lumped Mass & 50.0 & 35.6 & 64.4 \\
\hline $\bar{W}$ & $\begin{array}{l}\text { Structural } \\
\text { Weight }\end{array}$ & 136 & 125 & 148 \\
\hline$a$ & Acceleration & 28.1 & 20.2 & 39.1 \\
\hline$d$ & Displacement & -0.228 & -0.323 & -0.137 \\
\hline & $a_{\text {allowable }}$ & 29.0 & & \\
\hline & $d_{\text {allowable }}$ & -0.24 & & \\
\hline
\end{tabular}

The deterministic optimization, Problem A, is: minimize

$$
\begin{aligned}
& W \\
& a \leq a_{\text {allowable }} \\
& d \leq d_{\text {allowable }} \\
& v_{\mathrm{lb}} \leq v_{i} \leq v_{\mathrm{ub}} \\
& u_{j}=u_{\text {mean }}
\end{aligned}
$$

where $a$ is the maximum acceleration at the selected response location, $a_{\text {allowable }}$ is acceleration considered survivable, $d$ is the displacement of the floor beam at the monitoring station, and $d_{\text {allowable }}$ is the maximum displacement. For example, if the clearance between the floor beam and the fuselage is $d_{\text {allowable }}$, then this constraint discourages the optimization routine from operating with physically impossible displacements. The nondeterministic optimization, Problem B, is the same as A except that the constraint $d<d_{\text {allowable }}$ is replaced by a probabilistic constraint and each $u$ is replaced by a normal distribution.

Optimization Problems A and B were solved by using an approximate analysis to reduce the number of executions of the MSC.Dytran finite element code [4]. The formulation was implemented by using the iSIGHT commercial software package developed by Engineous Software, Inc. [5]. The iSIGHT software provides a reliability-based optimization approach where the optimization algorithm is the Modified Method of Feasible Directions, the UQ uses first-order reliability method (FORM), and the approximate analysis uses a kriging method.

Several commercial and public domain software packages were considered as alternatives to iSIGHT [2]. Of the candidates, one or two offered a better selection of UQ tools with better options for describing input distributions and diagnostic printed and graphic output. We chose iSIGHT because we needed optimization, UQ, and approximate analysis tools, and we did not find any other software with comparable capabilities. Other strengths of the iSIGHT package that are useful for aerospace applications include tools for task monitoring, integrating several disciplinary codes, saving input and outputs in a database, and experimenting with various optimization algorithms.

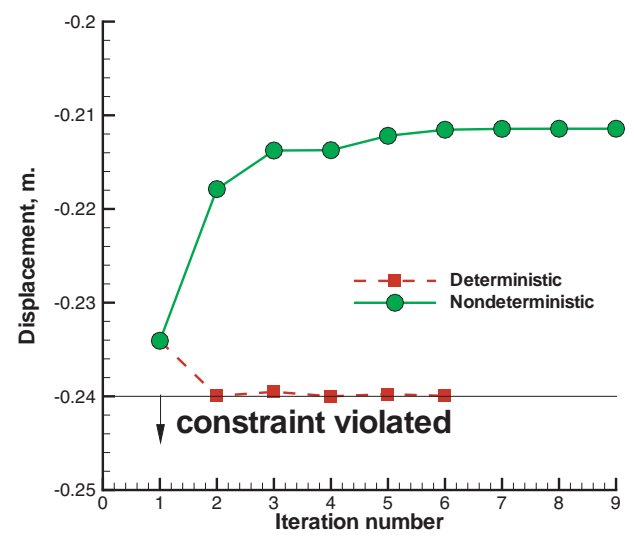

Figure 2. Impact dynamics optimization results

As seen in Fig. 2, Problem A converges to a solution with less structural weight $W$ and with a displacement $d$ that is exactly equal to $d_{\text {allowable }}$ Problem $\mathrm{B}$ converges to a solution with increased weight but with a greater probability that the calculated displacement is physically possible. At each of these solutions, approximate values of $\mathrm{W}, a$, and $d$ are confirmed by additional executions of the MSC.Dytran code. Moreover, the final probability of success measure is verified by using both FORM and Monte Carlo analyses in the iSIGHT package.

Reference [2] concludes that both Problems A and B have potential pitfalls. Problem A produces a design with low structural weight, but this design is adequate in only $50 \%$ of the crash scenarios. When mass $u_{3}$ or impact velocity $u_{2}$ is higher than the mean value, then the floor beam probably impacts the fuselage and the acceleration calculated by MSC.Dytran is invalid. Problem B produces a much more reliable design, but the analysis required for each iteration is more computationally expensive and the design space is more nonlinear; thus, Problem B requires more iterations of the optimization process.

Several lessons were learned from the impact dynamics optimization. First, using an approximate model for UQ and optimization is efficient and produces results with acceptable accuracy. However, the approximation is only valid over a relatively small range of design variable values. Thus, if any value of $v_{\mathrm{i}}$ changes too much, a new set of samples and a new approximate model will be required. Second, the computational cost of building the approximate model increases with the number of uncertain variables; thus, when a high-fidelity model is 
optimized, some variable screening technique is needed to decide which variables contribute to the uncertainty of the results. Finally, using optimization under uncertainty is a good way to avoid nonsensical optimization solutions. In this study, the probabilistic constraint successfully steers the algorithm away from solutions where FEM predicts a physically unrealistic response.

\section{Airfoil shape optimization}

The second example of optimization under uncertainty was studied because standard airfoil shape optimization procedures can generate noisy airfoils with off-design performance degradation [6]. A research initiative at NASA Langley investigated uncertainty-based optimization approaches for lift-constrained drag minimization in high-resolution design spaces. After three years of research, a winning strategy is evolving. The strategy is called the profile optimization method (POM) because it aims to reduce the drag profile over a range of flight conditions while keeping the lift at the target value.

A robust airfoil shape optimization over a transonic Mach range is demonstrated in [7]. Airfoil shapes are parameterized by B-splines, and a large number of vertical coordinates of the spline control points are used as design variables. Transonic airfoil performance is simulated for turbulent viscous flows in the Mach range from $M=0.68$ to $M=0.78$.

In general, robust optimization can be considered a multiobjective optimization with both the mean and variance of a performance measure as the objective functions. From this perspective, we can formulate the shape optimization over a Mach range as follows:

$$
\min \left(E\left(c_{d}\right), \sigma^{2}\left(c_{d}\right)\right)
$$

subject to:

$$
c_{l}(D, \alpha, M)=c_{l}^{*} \text { for } M_{\min } \leq M \leq M_{\max }
$$

where $c_{l}$ and $c_{d}$ are the lift and drag coefficients for a given airfoil, $D$ is the vector of design variables, $M$ is the free-stream Mach number, $c_{l}^{*}$ is the target lift value, $\alpha$ is the angle of attack needed to achieve $c_{l}^{*}$ at $M$, and $E\left(c_{d}\right)$ and $\sigma\left(c_{d}\right)$ are the mean and standard deviation of the drag coefficient with respect to $M$.

One could use any standard multiobjective optimization method to find a Pareto solution for Problem C. The drawback is that computational fluid dynamics (CFD) solutions for transonic turbulent viscous flows are computationally expensive. Thus, accurately predicting the mean and standard deviation of the drag with respect to a uniformly distributed random variable such as $M$ is impractical. A natural question is whether one could find reasonable approximations of robust solutions when reliable information on $E\left(c_{d}\right)$ and $\sigma\left(c_{d}\right)$ is not available. Our strategy is to use a few sample Mach points in the given Mach range as the design points. However, we do not use a standard numerical optimization procedure. Instead, we use the profile optimization procedure to reduce the drag simultaneously and proportionally at the design points, and consequently, reduce the drag profile over the whole Mach range. This process results in a simultaneous reduction of the mean and variance of the drag over the Mach range. This idea can be formalized by the following POM:

(1) Select design points $M_{1}, M_{2}, \ldots, M_{r}$.

(2) Evaluate the lift and drag and their gradients.

(3) Find a trust region size for a linear subproblem to achieve simultaneous and proportional drag reduction at all design points.

(4) Compute the least norm solution of the linear subproblem.

(5) Update the $D$ vector and repeat if necessary.

The linear subproblem mentioned in step (3) is formulated as follows:

subject to:

$$
\min _{\Delta D, \Delta \alpha_{i}} \max _{1 \leq i \leq r} \frac{c_{d}^{\text {predict }}\left(\Delta D, \Delta \alpha_{i}, M_{i}\right)}{c_{d}\left(D, \alpha_{i}, M_{i}\right)}
$$

$$
\begin{gathered}
c_{l}^{\text {predict }}\left(\Delta D, \Delta \alpha_{i}, M_{i}\right)=c_{l}^{*} \\
-\left|D_{i}\right| \delta \leq \Delta D \leq\left|D_{i}\right| \delta
\end{gathered}
$$

where $\delta$ is the scalar that controls the trust region size, $\Delta D$ is the change of design vector, $\Delta \alpha_{i}$ is the change of the angle of attack for $M_{\mathrm{i}}$, and $c_{d}^{\text {predict }}, c_{l}^{\text {predict }}$ are the linear predictions of the drag and lift corresponding to the modified airfoil shape, respectively. We choose the smallest $\delta$ such that the optimal objective function value of the above linear subproblem equals $(1-\gamma)$, where $\gamma$ is the specified drag reduction rate at the design points (ranging from $1 \%$ to $4 \%$ ).

The POM stated in Problem D is implemented with MPI parallel interface. The implemented research code does the following:

(1) uses $2 r$ computer nodes to compute the lift and drag and their gradients at $r$ design conditions in parallel (i.e., node $i$ computes the lift and its gradient at design condition $i$, and node $(r+i)$ computes the drag and its gradient at design condition $\mathrm{i}$, for $1 \leq i \leq \mathrm{r}$ );

(2) extracts geometry, function values, and gradient information from the outputs of the CFD code;

(3) formulates and solves the quadratic programming problem that produces the least norm solution of the linear subproblem mentioned in POM;

(4) updates the input geometry specification file; and

(5) repeats these steps for each iteration of POM. 
In [7], POM is tested on a realistic airfoil design by solving fully turbulent Navier-Stokes equations and the corresponding discrete adjoint equations. The initial RAE2822 airfoil is parameterized by $35 \mathrm{~B}$-spline control points, which allows a free-form deformation of the airfoil shape during the optimization process. The $x$ coordinates of the B-spline control points are fixed so that the chord length does not change, and the $y$-coordinates of the top and bottom surfaces are used as design variables. Additional geometry constraints are included so that the maximum airfoil thickness does not decrease, the thickness at two spar locations is controlled, and the five spline control points near the trailing edge can only change as a group. The design points are at $M=0.68$, $0.71,0.74$, and 0.76 , with $c_{l}^{*}=0.733$. The simultaneous and proportional drag reduction rate $\gamma$ is $3 \%$.

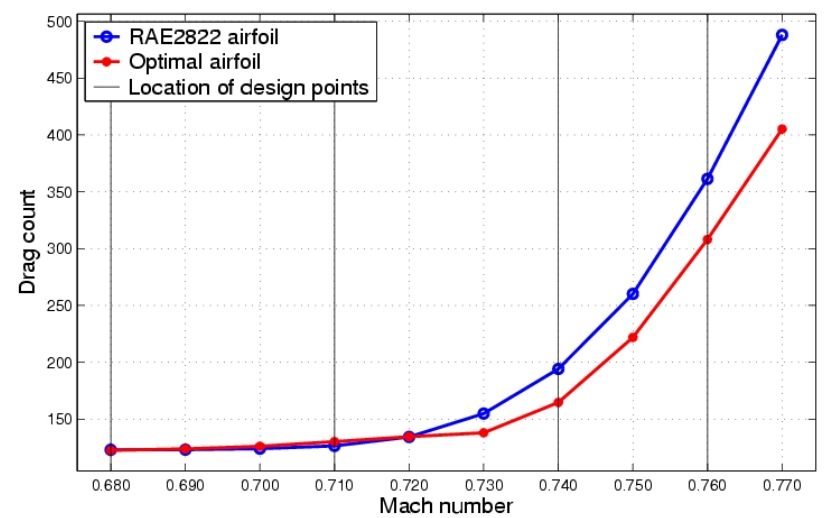

Figure 3. Typical drag reduction results for POM

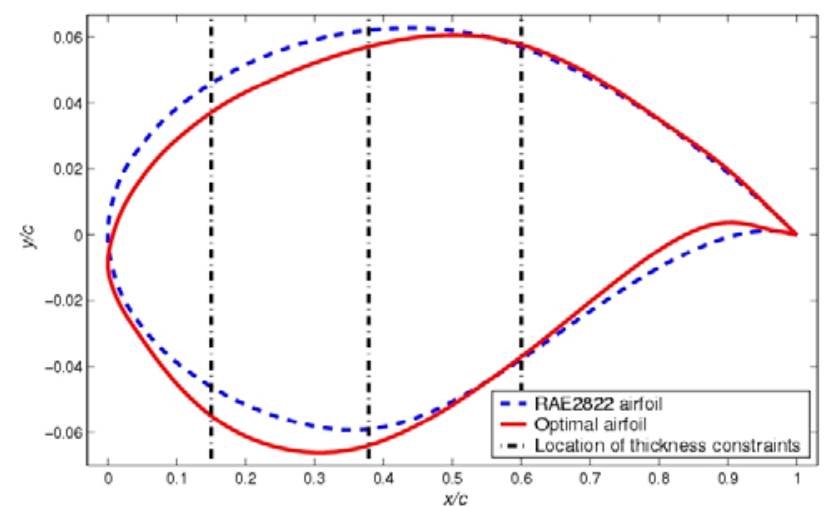

Figure 4. Comparison of RAE and optimal airfoils

Fig. 3 shows the drag profiles before and after 10 iterations of POM. Drag counts for the $10^{\text {th }}$ iterate are obviously less than those for the original airfoil although both airfoils provide the required lift. Fig. 3 shows that drag is reduced at the four design points. More significantly, Fig. 3 shows that drag is reduced at offdesign points that are not sampled during the optimization strategy. The improvement is greatest at the highest Mach number and more modest below $M=0.73$, which was the design Mach number for the original airfoil. Fig.
4 shows the difference in shape between the original RAE2822 airfoil and the optimal airfoil.

Several valuable lessons were learned during this research. First, POM can produce fairly realistic optimal airfoils. This robust optimization strategy helps to alleviate off-design performance degradation and the efficient shape modification leads to fairly smooth optimal airfoil shapes without airfoil smoothing. Second, it is important to have relatively accurate gradients of the lift and drag coefficients so that the optimizer is not adversely affected by the noise in the gradients. Noisy gradients tend to lead to unrealistic and random changes of the airfoil shape. Once a bump is created on the airfoil, the optimizer does not have the ability to remove the bump, and it tends to make the airfoil shape more oscillatory in the following iterations. Third, to make POM a useful tool in real-world design environments, we need to incorporate airfoil smoothing, develop more flexible drag reduction strategies while enforcing robust optimization policy, use more flexible thickness constraints that allow thickness locations to change during the optimization process, and demonstrate the feasibility of POM for 3-D wing design.

\section{Aerodynamic/structural wing design}

The last example of optimization under uncertainty aims to improve the performance of a 3-D flexible wing while taking into account the coupling between the aerodynamic loads and the structural deformation. Geometric uncertainty is included by using a first-order second moment (FOSM) statistical approximation to propagate the input uncertainty through the coupled CFD and FEM codes.

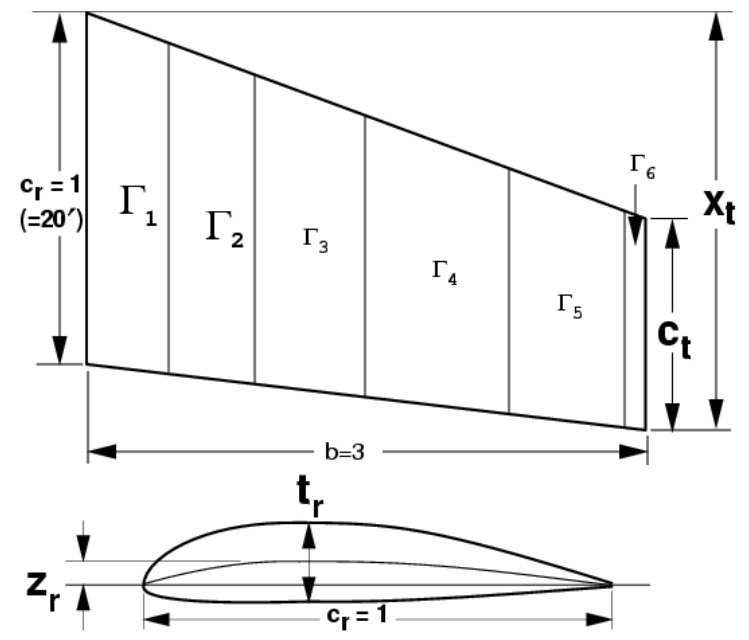

Figure 5. Potential 3-D wing design variables

Fig. 5 shows the geometry of the 3-D wing and indicates some potential design variables. The traditional aerodynamic variables are the airfoil shape and the wing 
planform shape; the traditional structural variables are the skin thickness, web thickness and truss cross-sectional areas in each of six zones (i.e., $\Gamma_{1}-\Gamma_{6}$ ). Four design variables are identified as being particularly sensitive to anticipated levels of manufacturing uncertainty. These are $t_{\mathrm{r}}$ and $z_{\mathrm{r}}$, the maximum thickness and camber of the root airfoil section, and $\Gamma_{1}$ and $\Gamma_{2}$, the structural sizing factor for the two zones near the root. To compare optimization with and without uncertainty, we assume the four design variables are statistically independent and normally distributed. For the cases discussed here, a coefficient of variation equal to 0.0001 was chosen for all design variables.

The optimization problem is stated as:

$$
\begin{array}{lc}
\text { Minimize: } & -(L / D)^{2} \\
\text { subject to: } & \\
& g_{j}+k \sigma_{j} \leq 0 \quad \text { for } j=1,2,3
\end{array}
$$

where $L / D$ is the lift to drag ratio of the wing, $g_{j}$ is the $j$ th inequality constraint evaluated at the mean of each design variable, $\sigma_{j}$ is the approximate standard deviation of $g_{j}$, and $k$ is a user-defined parameter that adjusts each constraint to achieve a desired probability of satisfaction. The objective function is representative of design cruise conditions for a transport aircraft. The three constraints are probabilistic constraints on payload, trim, and compliance requirements. In particular, $g_{1}$ is a lower limit on payload (i.e., the difference between the total lift and the structural weight), $g_{2}$ is a limit on trim difficulty (i.e., the wing pitching moment that can be offset by movement of the tail surfaces), and $g_{3}$ is an upper limit on compliance (i.e., the work performed by the aerodynamic loads to deflect the structure). Bounds on the design variables and purely geometric constraints on the leadingedge radius are also added to Problem E.

One challenging aspect of this research involves integration of disciplinary codes to create the coupled analysis. Reference [8] has a description of this process and provides important references. The disciplinary codes are executed by a separate driver program and by UNIX scripts. Each code runs independently, some simultaneously on separate processors, and the required data transfers between them, also directed by the driver, are accomplished via data files.

The CFD code used for aerodynamic sensitivity analysis was generated by applying the automatic differentiation code ADIFOR to produce a relatively efficient, direct mode, gradient analysis code [9]. The surface geometry, volume mesh and structural analysis codes were also preprocessed with ADIFOR. The structural analysis code used to compute the deflection of the elastic wing was a generic finite element code. Because the elastic deformation was assumed to be small, linear elasticity was deemed to be appropriate. At the wing surface (i.e., the interface where aerodynamic load and structural deflection information is transferred), nodes of the FEM were assumed to be a subset of the CFD aerodynamic surface mesh points. This choice allowed for simplifications in the data transfers and was deemed suitable for these initial 3-D robust optimization demonstrations.

A deterministic optimization was performed first by setting $k=0$ in Problem E. That deterministic result was used as the initial design for all robust optimizations. The deterministic optimization process reduced the airfoil section thickness $t_{\mathrm{r}}$ to reduce the shock strength, thereby reducing the drag and improving the $L / D$. Consequently, the thinner wing became more flexible, which allowed the tip to twist and increased the magnitude of the pitching moment. To satisfy the trim constraint, the structural element thickness increased; consequently, the wing became heavier. To satisfy the payload constraint, the section camber increased. All these changes in design affect the wing bending but the compliance constraint is never active.

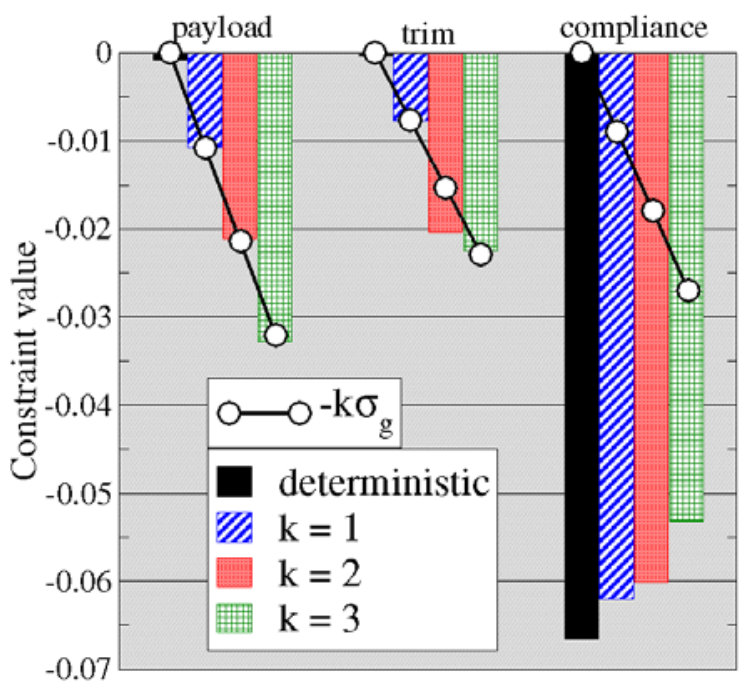

\section{Figure 6. Change in constraint values with increased uncertainty in geometric variables}

Fig. 6 presents the results for the deterministic and robust optimizations for several values of $k$. An increase of the parameter $k$ can be interpreted as an increase in the specified minimum probability that the constraints are satisfied with respect to random inputs. For example, if the output responses have a normal Gaussian distribution and $k=3$, then $g_{j}+3 \sigma_{j} \leq 0$ implies that there is at least a $99.87 \%$ probability that each $g_{j} \leq 0$ constraint is satisfied. For $k=1$ and $k=2$, the corresponding minimum probabilities are $84.13 \%$ and $97.73 \%$.

Changes in the constraint boundaries due to changes in $k$ are indicated by circles in Fig. 6. Mean values of the constraint function greater (less negative) than each circle would indicate violated robust constraints; that is, the probability of the constraint being satisfied would be less 
than the specified minimum probability. Seemingly small changes in minimum probabilities produce substantial changes in the normalized constraint values, which must be accounted for in the optimization. The effect on the design variable values is substantial, but the effect on the objective function, and consequently the $L / D$, is rather small. As with the robust airfoil shape optimization study, it appears that many wing shapes will perform well; robust optimization attempts to find that design which performs well assuming manufacturing uncertainty.

We learned several lessons while studying the effect of uncertainty on the design of a flexible wing in transonic flow. We conclude that a statistical FOSM method is appropriate to propagate the input uncertainties through the multidisciplinary analyses (e.g., CFD for aerodynamics and FEM for structures) to determine effects on output parameters. Reference [10] used several reliability assessment methods to check the accuracy of the approximate results in [8]. The first derivatives required for the FOSM method can be obtained from automatic differentiation of the individual codes used in the analysis. This implementation of the statistical approach is easy to retrofit into gradient-based design codes that already use analytical or semianalytical sensitivity derivatives for optimization. However, our numerical studies also suggest that increased input uncertainty or increased reliability requirements have a significant effect on the number of active constraints and on the size and shape of the feasible region of design space [8]. In an extreme case, the feasible region can disappear, and it becomes difficult to judge whether the results of the optimization process are useful or not. The fact that a deterministic solution exists is no guarantee that a solution exists for the robust optimization problem.

\section{Concluding remarks}

This paper briefly describes three aerospace applications of optimization under uncertainty. Whereas [1] outlines the need for all UBM research, this paper focuses on practical implementation issues for robust or reliabilitybased optimization. Our experience with the three applications and our study of the optimization literature leads us to make several recommendations for further research. We see the need for:

(1) Approximation methods for combining expensive physics-based analysis with UBM.

(2) Multiobjective optimization methods that find designs with the highest probability of success even if there is no feasible design space.

(3) Efficient and validated UBM for coupled, multidisciplinary analysis.

(4) Methods for including physics-based uncertainty quantification in conceptual design and system risk assessments.
(5) Ways to exploit multiple-fidelity models to reduce the computational expense of UBM.

(6) UBM for time-dependant analyses like impact dynamics.

This prioritized list of enabling technologies represents the authors' views of the most pressing issues for application of uncertainty-based methods to aerospace vehicle design.

\section{References}

[1] T.A. Zang, et al., "Needs and Opportunities for UncertaintyBased Multidisciplinary Design Methods for Aerospace Vehicles", NASA-TM-2002-211462.

[2] K. Lyle, S. Padula, and A. Stockwell, "Applications of Probabilistic Analysis to Aircraft Impact Dynamics", AIAA2003-1482.

[3] K. Lyle, A. Stockwell, and R. Hardy, "Applications of Probability Method to Assess Crash Modeling Uncertainty", AHS Forum, Phoenix, AZ, May 6-8, 2003.

[4] Anon., "MSC.Dytran User's Manual", Version 2002, Santa Ana, CA, Nov. 2001.

[5] Anon., "iSIGHT Reference Guide", Version 7.0, Engineous Software, Inc., Morrisville, NC, 2002.

[6] S. Padula, and W. Li., "Options for Robust Airfoil Optimization Under Uncertainty”, AIAA-2002-5602.

[7] W. Li, and S. Padula, "Performance Trades Study for Robust Airfoil Shape Optimization", AIAA-2003-3790.

[8] C. Gumbert, P. Newman, and G. Hou, "Effect of Random Geometric Uncertainty on the Computational Design of a 3-D Flexible Wing", AIAA-2002-2806.

[9] C. Bischof, A. Carle, P. Khademi, and A. Mauer, "Automatic Differentiation of FORTRAN", IEEE Comp. Sci. and Eng., 3(3), 1996, pp. 18-32.

[10] C. Gumbert, G. Hou, and P. Newman, "Reliability Assessment of a Robust Design Under Uncertainty for a 3-D Flexible Wing”, AIAA-2003-4094. 